\title{
Design of a Microwave Lowpass - Bandpass Filter using Deep Learning and Artificial Intelligence
}

\author{
Saeed Roshani, Hossein Heshmati, Sobhan Roshani* \\ Department of Electrical Engineering, Kermanshah Branch, Islamic Azad University, Kermanshah, Iran \\ *Corresponding Author: Sobhan Roshani, Email: s.roshani@aut.ac.ir
}

How to cite this paper: Saeed Roshani, Hossein Heshmati, Sobhan Roshani (2021). Design of a Microwave Lowpass - Bandpass Filter using Deep Learning and Artificial Intelligence. Journal of the Institute of Electronics and Computer, 3, 1-16. https://doi.org/10.33969/JIEC.2021.31001

Received: June 25, 2021

Accepted: July 30, 2021

Published: August 9, 2021

Copyright (C) 2021 by author(s) and Institute of Electronics and Computer. This work is licensed under the Creative Commons Attribution International License (CC BY 4.0).

http://creativecommons.org/licenses/by/4.0/

\begin{abstract}
In this paper, a lowpass - bandpass dual band microwave filter is designed by using deep learning and artificial intelligence. The designed filter has compact size and desirable pass bands. In the proposed filter, the resonators with Z-shaped and T-shaped lines are used to design the low pass channel, while coupling lines, stepped impedance resonators and open ended stubs are utilized to design the bandpass channel. Artificial neural network (ANN) and deep learning (DL) technique has been utilized to extract the proposed filter transfer function, so the values of the transmission zeros can be located in the desired frequency. This technique can also be used for the other electrical devices. The lowpass channel cut off frequency is $1 \mathrm{GHz}$, with better than $0.2 \mathrm{~dB}$ insertion loss. Also, the bandpass channel main frequency is designed at $2.4 \mathrm{GHz}$ with $0.5 \mathrm{~dB}$ insertion loss in the passband.
\end{abstract}

\section{Keywords}

Artificial neural network (ANN), deep learning (DL), dual band, lowpass - bandpass, microwave filter

\section{Introduction}

Microstrip structures are widely used in microwave devices. Microwave filter is basic device which can be used to design the other microwave devices, such as diplexers [1-2], power dividers [3-4], power amplifiers [5-6] and several other devices for mobile and wireless communications systems [7]. In communication applications, sometimes it is necessary to separate the lowpass frequencies from the higher frequencies. Therefore, the multiband filters and diplexers are important in this application. Subsequently, the dual band lowpass-bandpass filter would be desirable in the modern communication systems applications. In previous works, 
several dual band filters have been designed, but they suffer from poor parameters, such as large size, undesirable return loss, and undesirable insertion loss.

In [8], a dual band filter is designed using open ended stubs and diodes. However, in this filter, the insertion loss is high in both lowpass and bandpass channels. In [9], a multi band filter is presented by using two quarter wave length lines and open ended stubs. There is a lowpass channel and two bandpass channels in the designed filter in [9], but the insertion loss in channels are very high. For example, there is $1.9 \mathrm{~dB}$ insertion loss in one of the bandpass channels, which is not acceptable.

A multi band filter with one lowpass channel and two band pass channels are presented in [10]. In this filter, stepped resonators are utilized to sharpen the transition band in the lowpass channel. In the designed filter in [10], the lowpass channel cutoff frequency is $1 \mathrm{GHz}$ with $0.8 \mathrm{~dB}$ insertion loss, and the bandpass channels are at 2.4 and $5.8 \mathrm{GHz}$ with 2.1 and $2.5 \mathrm{~dB}$ insertion loss, respectively. According to the results in [10], the obtained insertion losses are not acceptable, which mean large amount of power will be lost in the channels of this filter.

Besides, neural networks, which are useful tools in solving the engineering problems have been recently used to model the filters [11-15]. In [16] back propagation ANN is utilized for designing of a microstrip filter. Stepped impedance resonator and open stub lines are used to design the lowpass filter in [16]

In this paper, a dual band lowpass-bandpass filter is designed by utilizing the deep learning and artificial intelligence techniques. The basic structure of the filter is adopted from a diplexer structures designed by authors in [17]. The designed operating frequency are applicable frequencies which can be used in Wi-Fi and 5G applications.

\section{Design of the Double Band Lowpass-Bandpass Filter}

The designed lowpass-bandpass filter is composed of a lowpass part and a bandpass part. The presented filter is designed on a RO4003C substrate with $0.813 \mathrm{~mm}$ thickness and 3.38 of permittivity.

\subsection{Low Pass Filter Design}

To design the final lowpass-bandpass filter, at first, the lowpass filter (LPF) is designed. In design of the lowpass filter, bandwidth, transition band sharpness and transmission zeros are important factors. To design of the lowpass filter, firstly, a resonator with desirable response is presented. The LC equivalent circuit (LCEC) of the primary lowpass resonator is depicted in Figure 1. Also, LCEC frequency response of the proposed primary resonator is shown in Figure 2. The values of the utilized inductors and capacitors in the LCEC of the primary lowpass resonator are 
listed in Table 1.

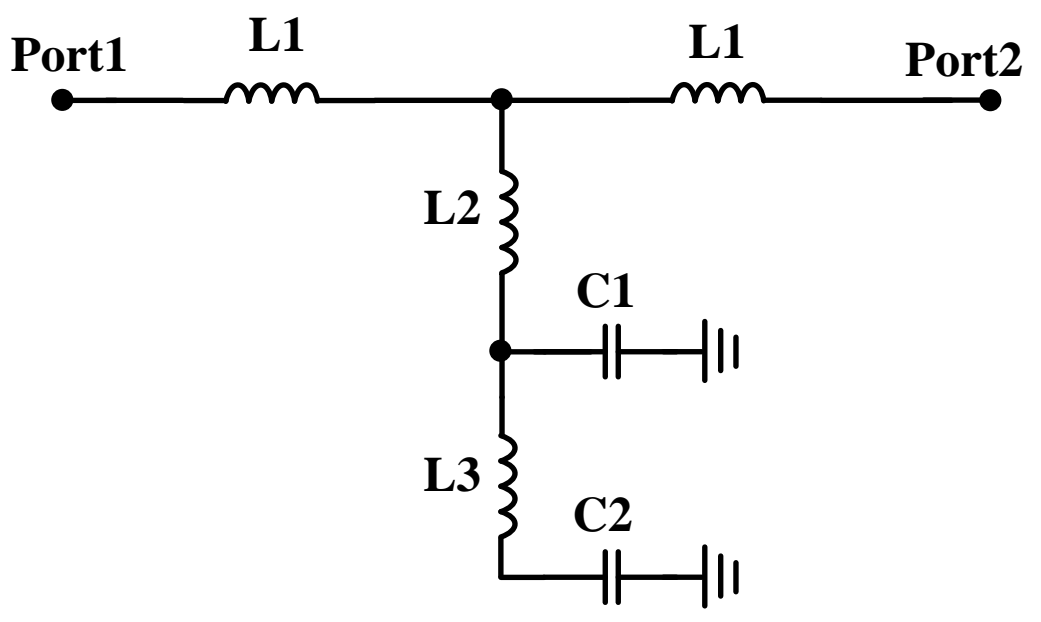

Figure 1. The LCEC of the primary lowpass resonator.

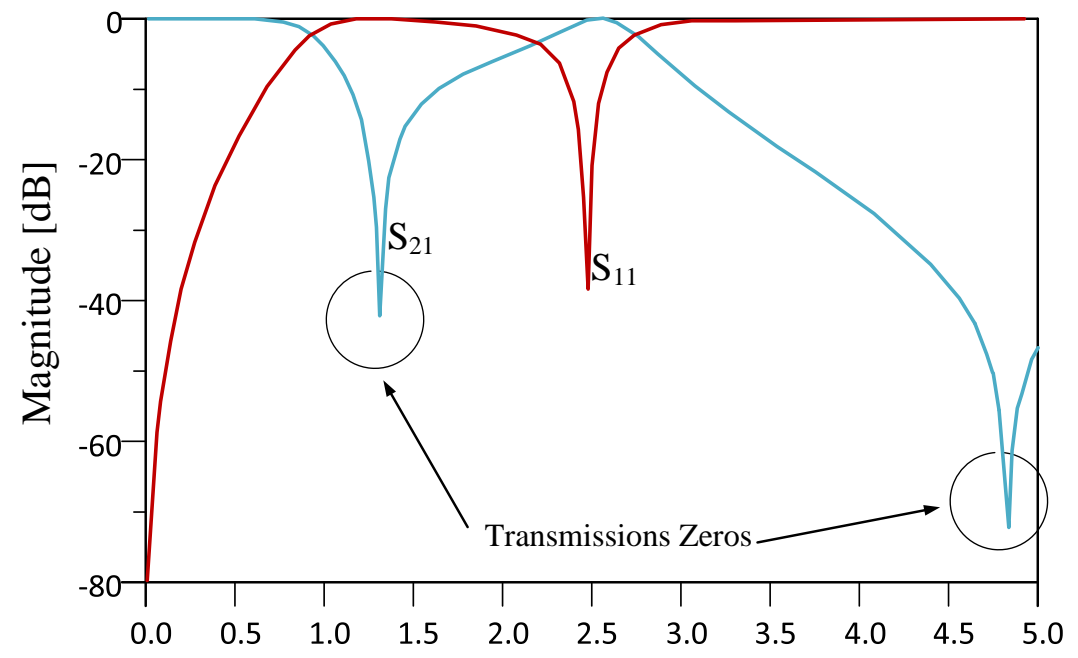

Figure 2. The LCEC frequency response of the proposed primary lowpass resonator

Table 1. The values of the utilized inductors and capacitors in the LC circuit of the primary lowpass resonator.

\begin{tabular}{ccc}
\hline $\mathrm{L} 1=5.4 \mathrm{nH}$ & $\mathrm{L} 2=0.6 \mathrm{nH}$ & $\mathrm{L} 3=5.5 \mathrm{nH}$ \\
\hline $\mathrm{C} 1=2 \mathrm{pF}$ & $\mathrm{C} 2=2.3 \mathrm{pF}$ & \\
\hline
\end{tabular}




\subsection{Artificial Intelligence and Design of the Lowpass Filter}

After designing the primary lowpass resonator, in the next step the artificial neural network (ANN) and deep learning (DL) is utilized to design the lowpass filer. The ANN and DL are used to extract the transfer function of the primary resonator, so the values of the transmission zeros can be located in the desired frequency. In this paper multilayer perceptron (MLP) neural network with several layers is considered for deep learning.

The transfer function of the presented LC circuit can be used to identify the resonator behaviors, such as transmission zeros locations. However, the extracting of the transfer function is a complex procedure even impossible in some cases. Therefore, ANN and DL tools are used to model the transfer function of the LC circuit of the primary resonator. The structure of proposed ANN model is shown in Figure 3, which consists of an input, two hidden and an output layers. Also, the block diagram of the designed neural model is shown in Figure 4.

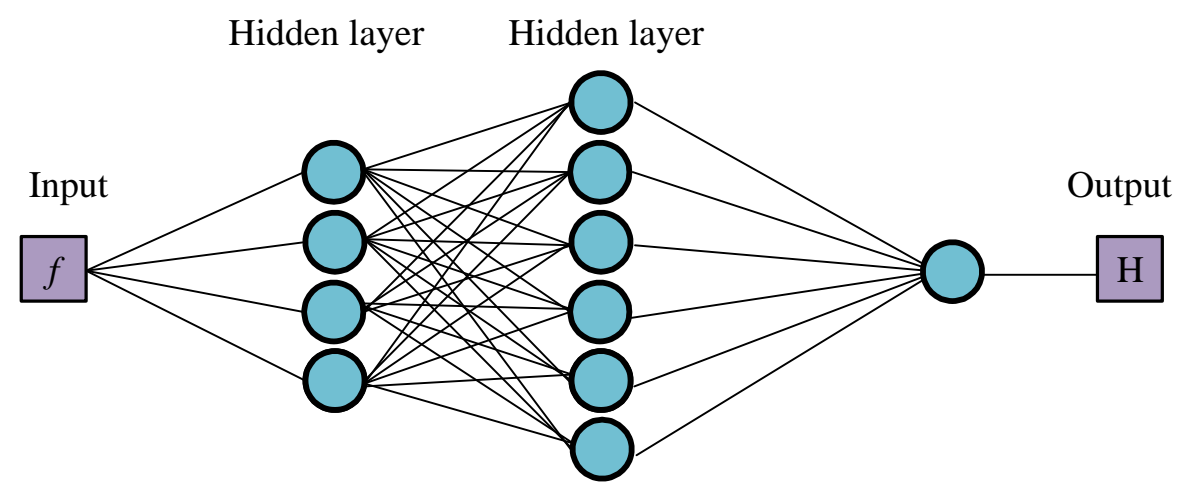

Figure 3. The structure of proposed ANN model.

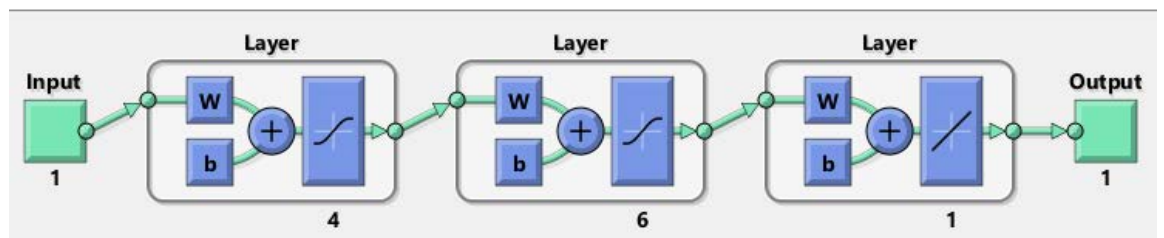

Figure 4. The block diagram of the designed neural model.

The estimated and real resonator transfer function is depicted in Figure 5. As can be seen in Figure 5, the proposed model can precisely estimate the transfer function of the resonator. The results show that the root mean square errors (RMSE) of the designed ANN model are 0.013 and 0.017, respectively which show high precision of the proposed model. 


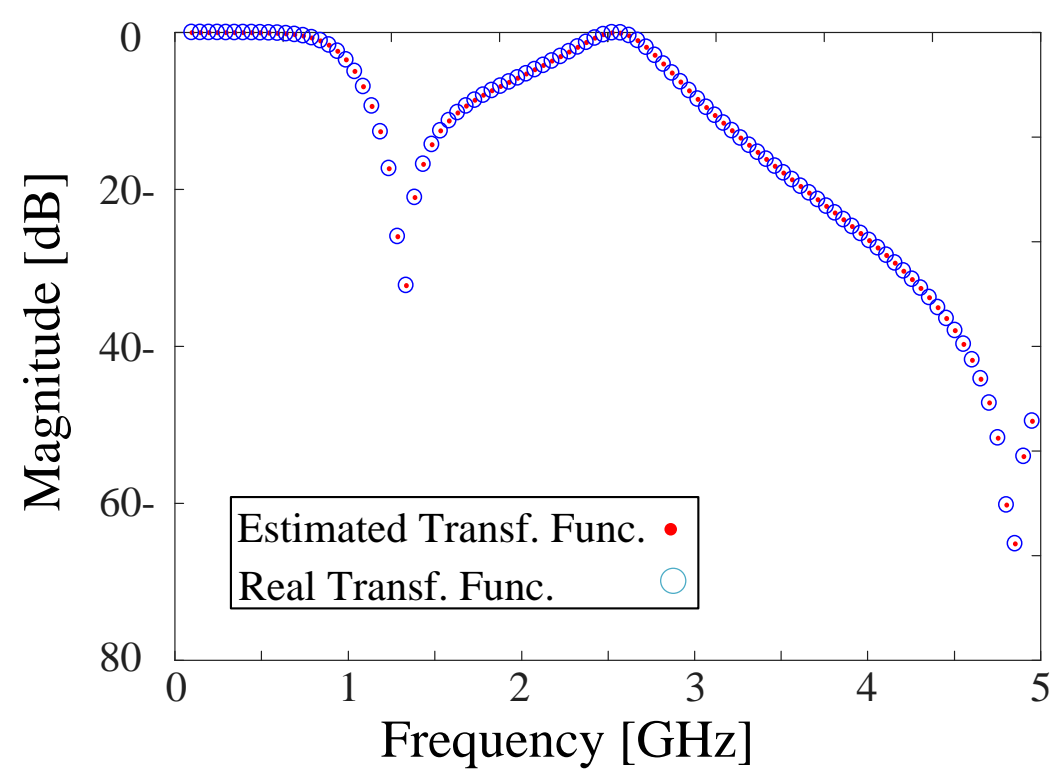

Figure 5. The estimated and real the transfer function of the resonator.

The obtained regression results for training and testing procedures of the designed neural model are illustrated in Figure 6. As seen, the designed ANN model has precisely modeled the transfer function of the primary resonator.

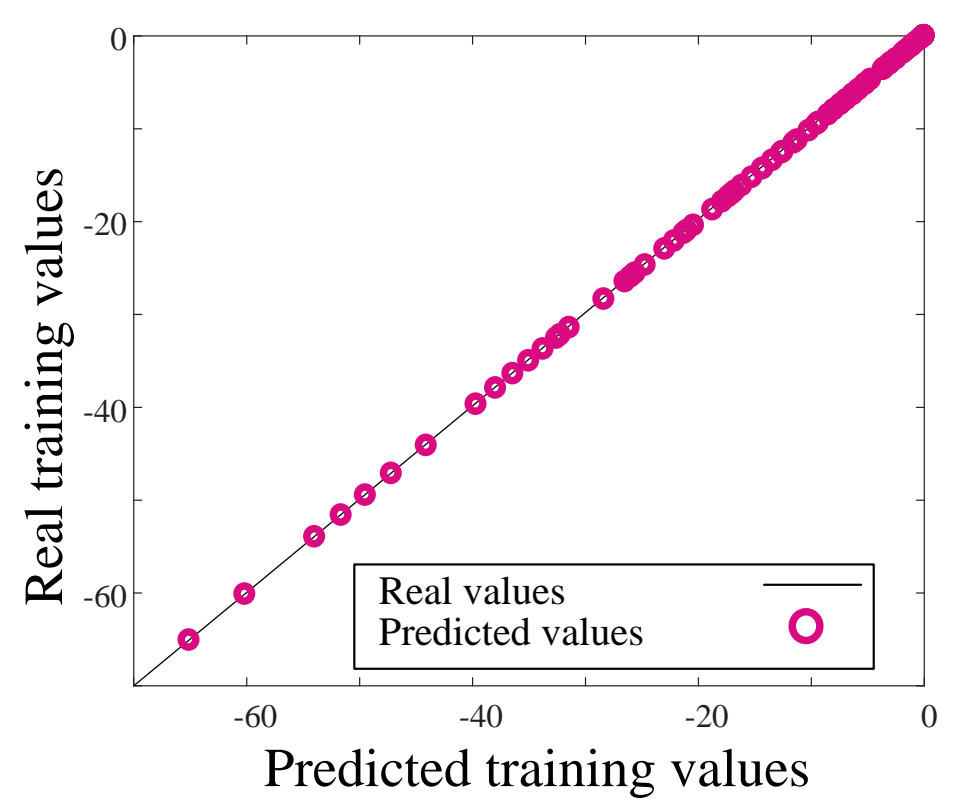

(a) 


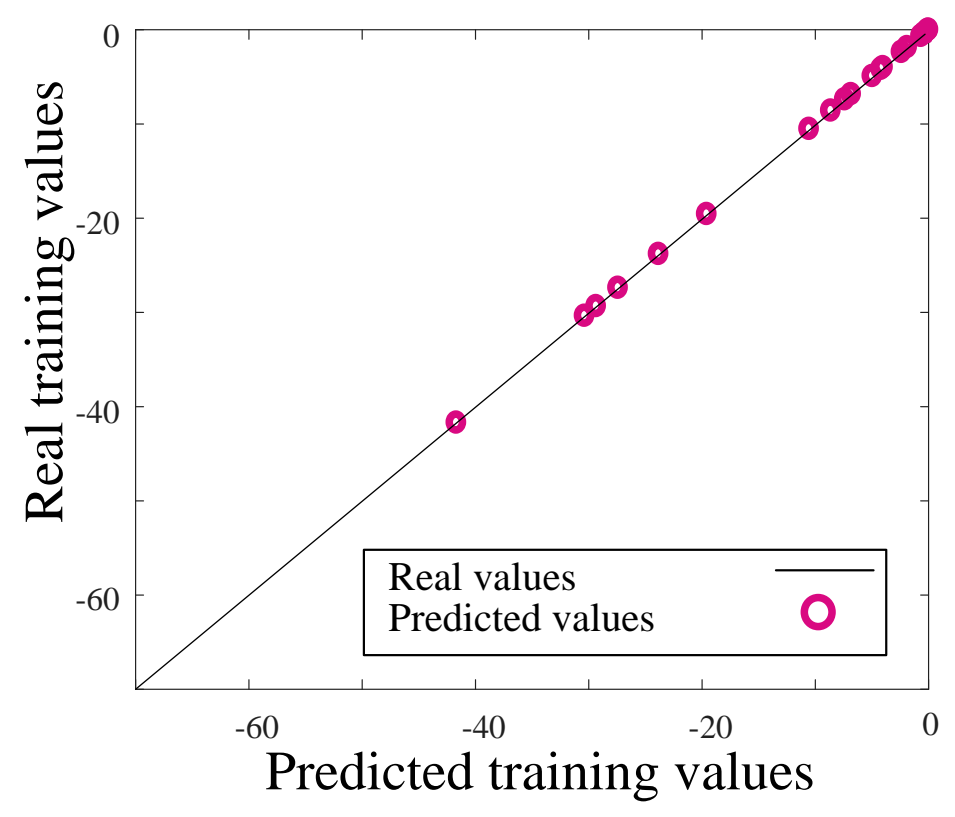

(b)

Figure 6. The regression results for training and testing steps of the the designed neural model.

\subsection{Lowpass Filer Design}

According to the obtained transfer function by using the ANNs, the transmission line realization of the proposed primary resonator is designed. The frequency response and layout of the proposed primary resonator are shown in Figure 7(a). As seen, the dash line curve shows LC circuit model response and solid line shows the layout electromagnetics simulation (EM simulation) of the primary resonator. According to the results, the obtained frequency response of the primary resonator layout verifies the primary resonator transfer function. Also, the obtained transmission zeros are located at desired frequencies. The effects of parameter "d (mm)" on the response of the primary resonator is shown in Figure 7(b). 
Saeed Roshani et al.

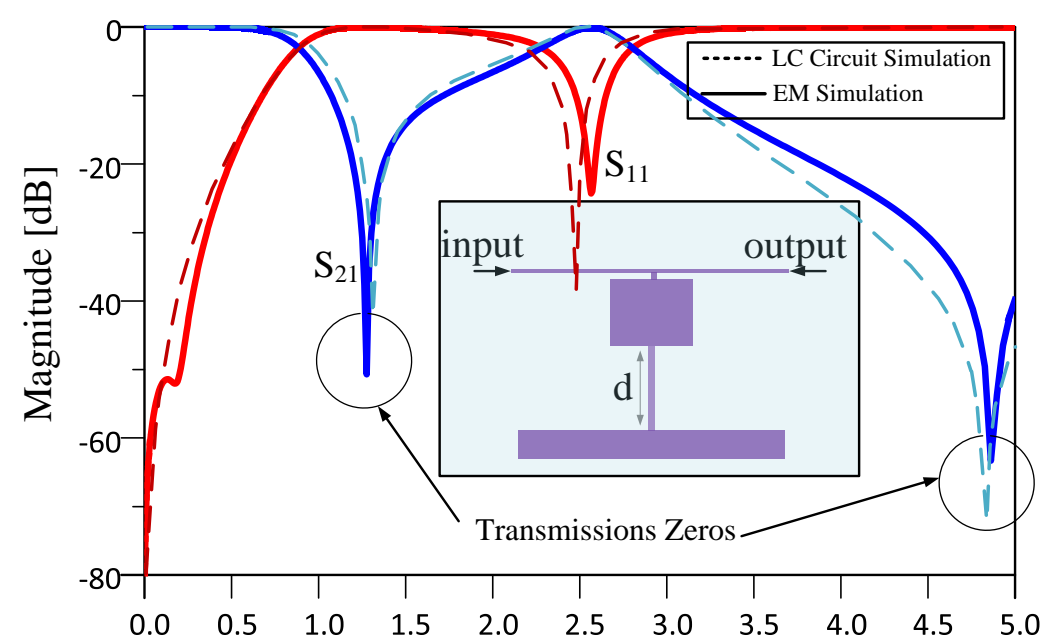

(a)

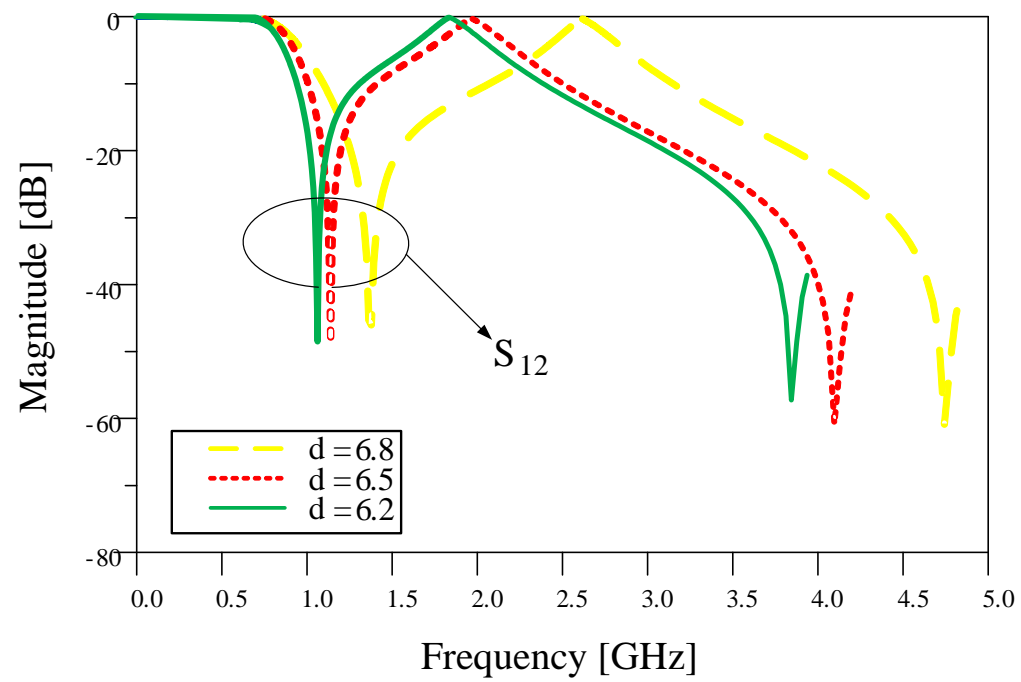

(b)

Figure 7. (a) The frequency response and layout of the proposed primary resonator (b) Effects of parameter "d (mm)" on the response of the primary resonator.

Then, the Z-shaped resonators are designed similar to the primary resonator design procedures. These two Z-shaped resonators improve the suppression band and also sharpen the transition band of the lowpass filter. The frequency response and layout of the proposed Z-shaped resonator are illustrated in Figure 8. 


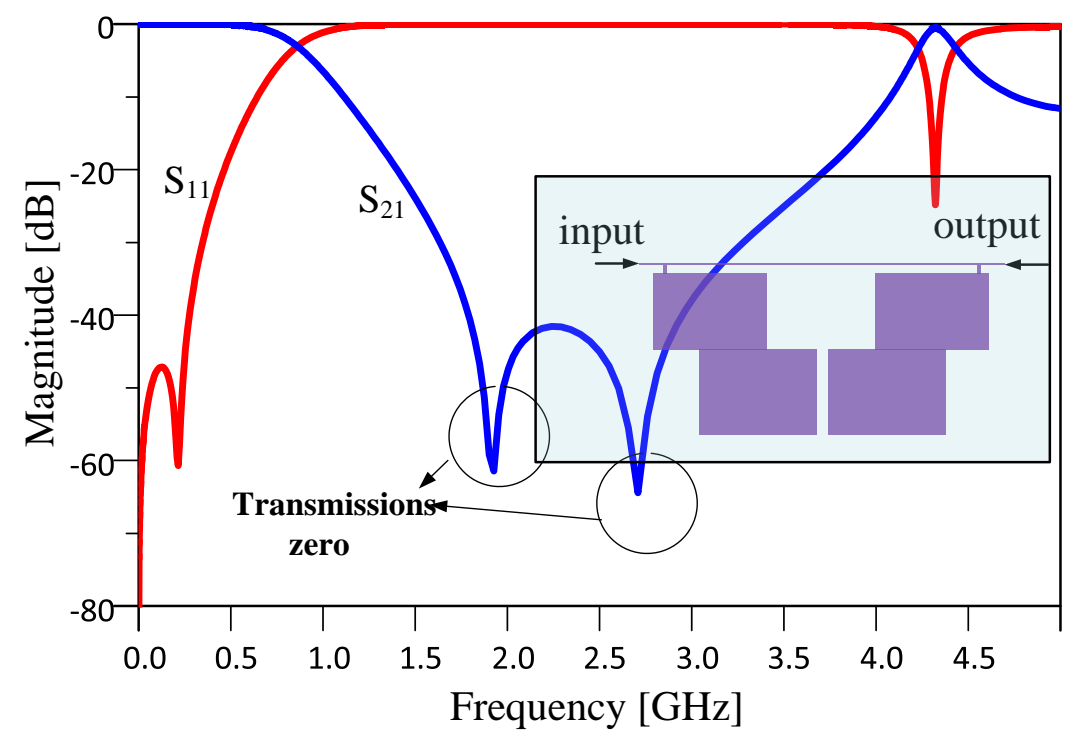

Figure 8. The frequency response and layout of the proposed Z-shaped resonator.

The lowpass filter is obtained by combining the designed primary resonator and Z-shaped resonator. The frequency response and layout of the primary lowpass filter are illustrated in Figure 9. As seen, the obtained suppression band is not very wide, so in the next step, two suppressers are added in the layout to form the proposed low pass filter.

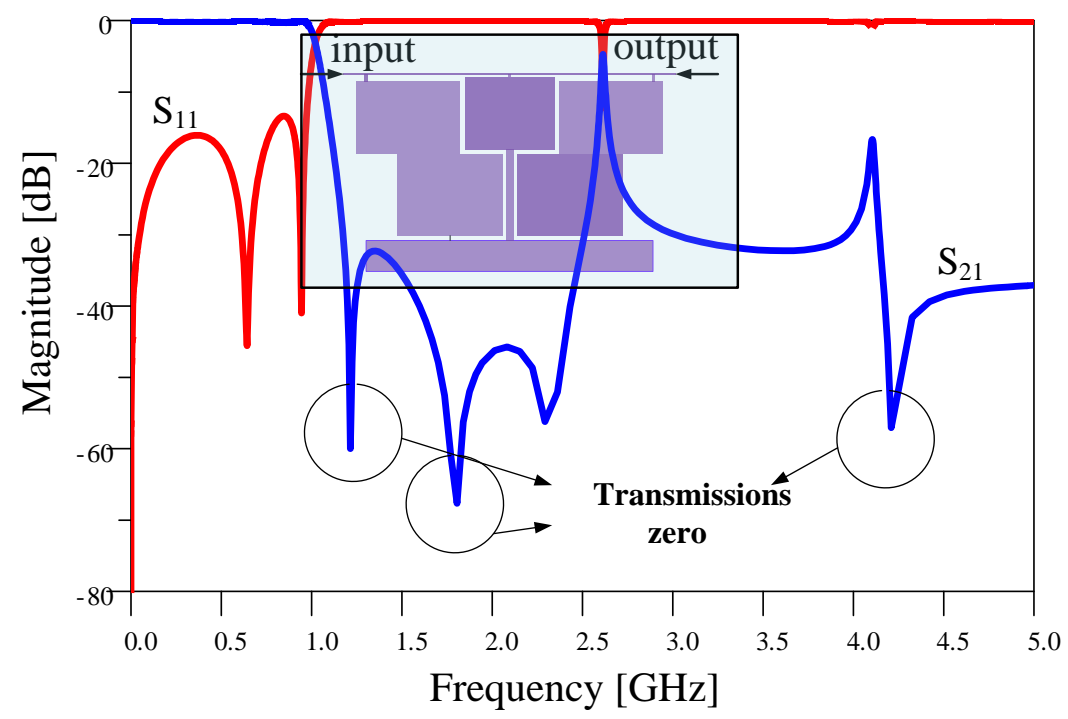

Figure 9. The frequency response and layout of the primary lowpass filter. 
The frequency response and layout of the final proposed lowpass filter is shown in Figure 10. As can be seen in this figure, the proposed low pass filter has a very wide suppression band, sharp transition band and flat passband, which are desirable factors for a lowpass filter.

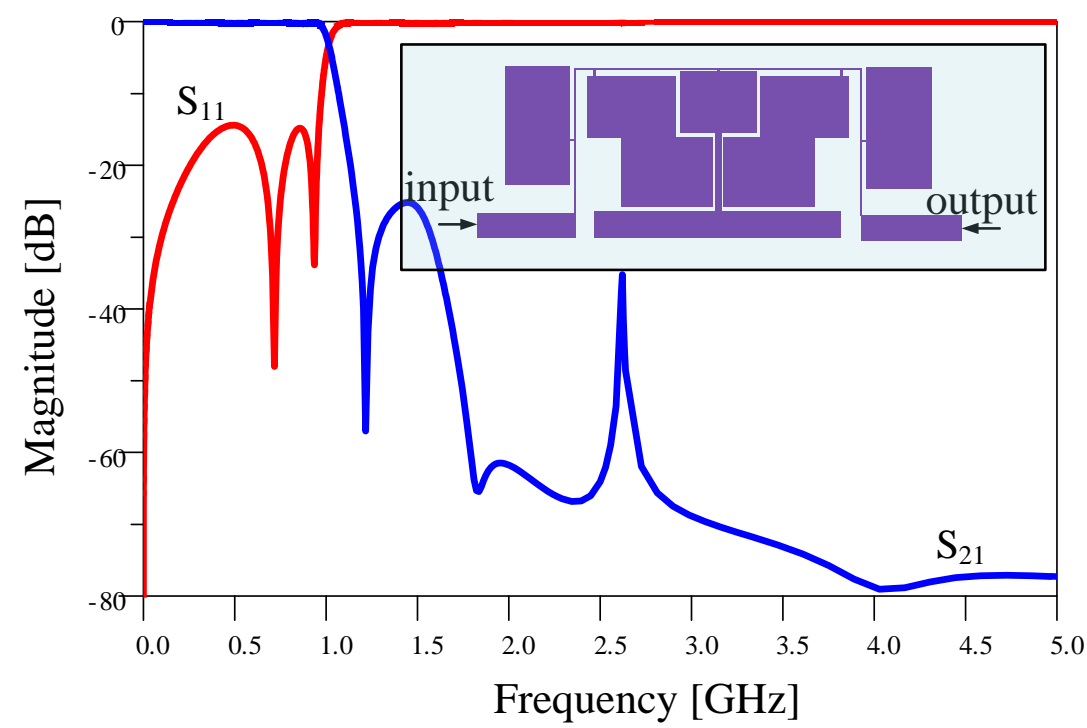

Figure 10. The frequency response and layout of the proposed lowpass filter.

\subsection{Design of the Bandpass Filter}

Designing of the bandpass filter (BPF) is an important step to design the dual band filter. The fundamental frequency of the BPF should be precisely located at the desired frequency. To design the BPF at the desired frequency, a resonator composed of two coupled open ended stubs is used as shown in Figure 11. The frequency response and layout of the primary BPF are depicted in Figure 11. The desired operating frequency can be achieved by tuning the lengths of the couple lines and the gap between the coupled lines. The result shows that the desired main frequency of $2.4 \mathrm{GHz}$ is correctly achieved for the coupled lines resonator 


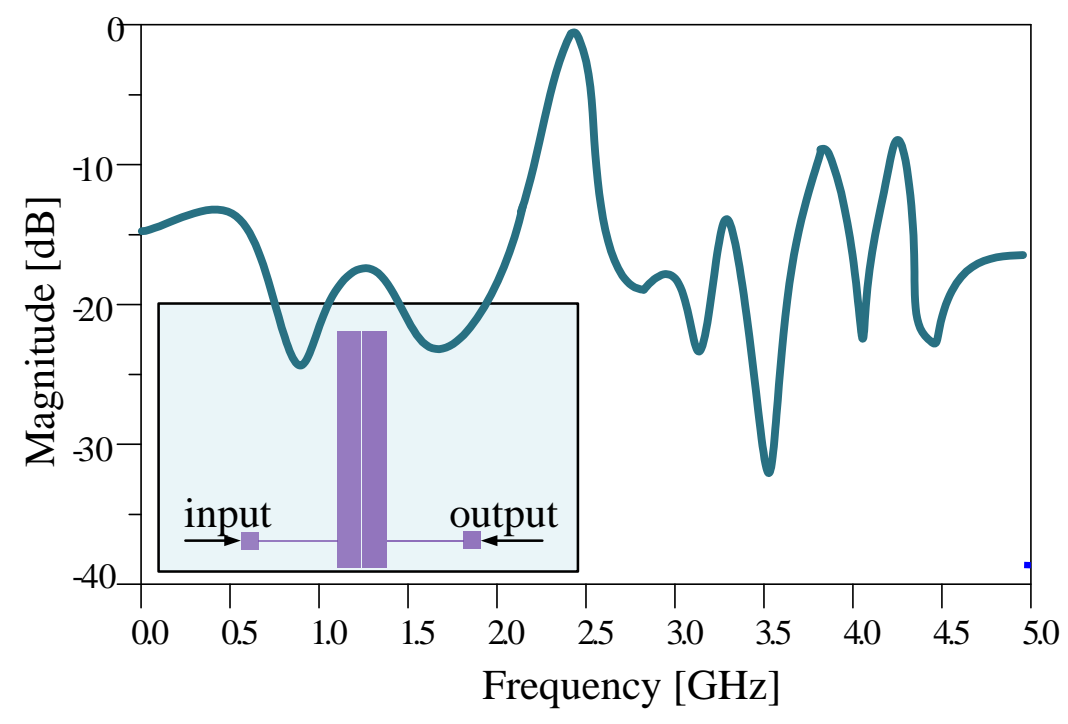

Figure 11. The frequency response and layout of the primary bandpass filter.

\subsection{Design of the Proposed Filter}

By combining the designed lowpass and bandpass filters in the previous stages, the double band lowpass-bandpass filter will be formed, which can be used in mobile and wireless communication systems. The layout and frequency response of the proposed dual band lowpass-bandpass filter are illustrated in Figures 12 and 13. Dimensions of the utilized transmission lines in the proposed filter are listed in Table 2. 
Saeed Roshani et al.

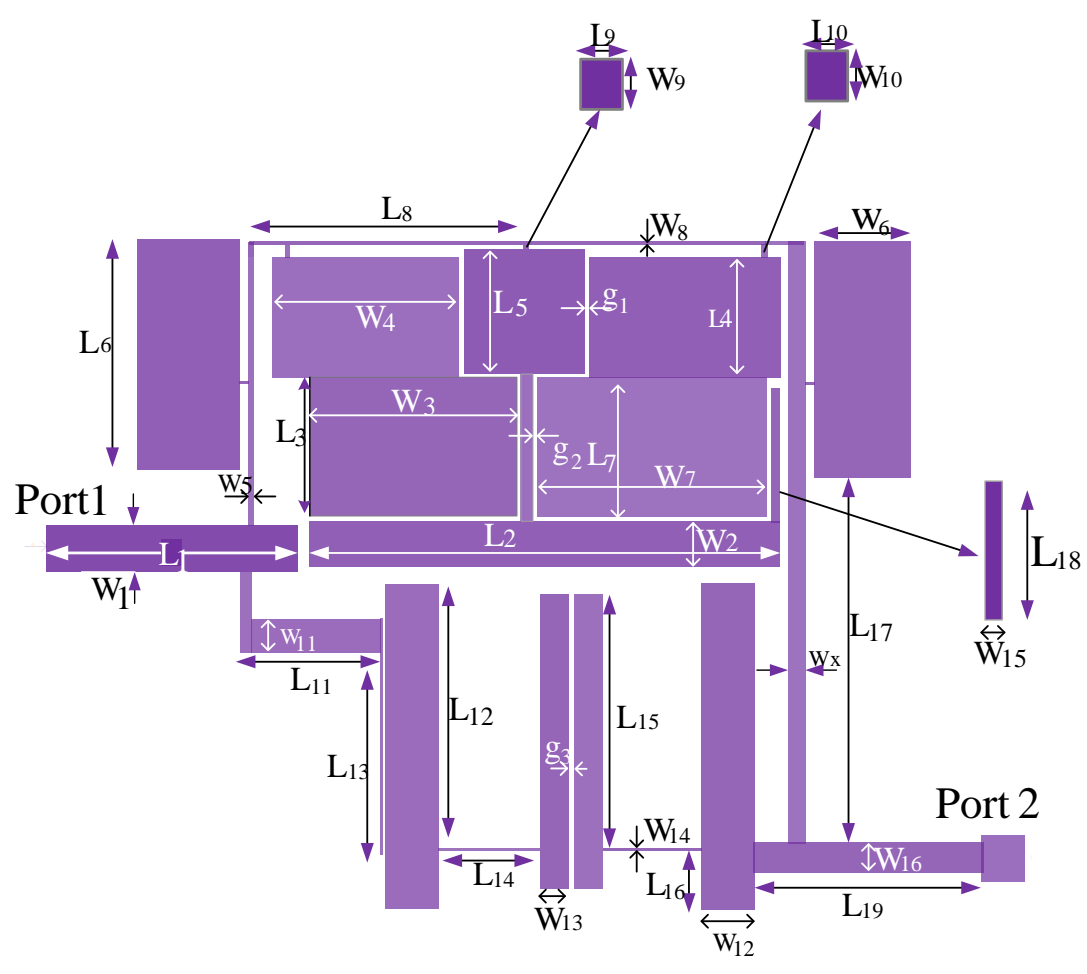

Figure 12. Layout of the proposed filter.

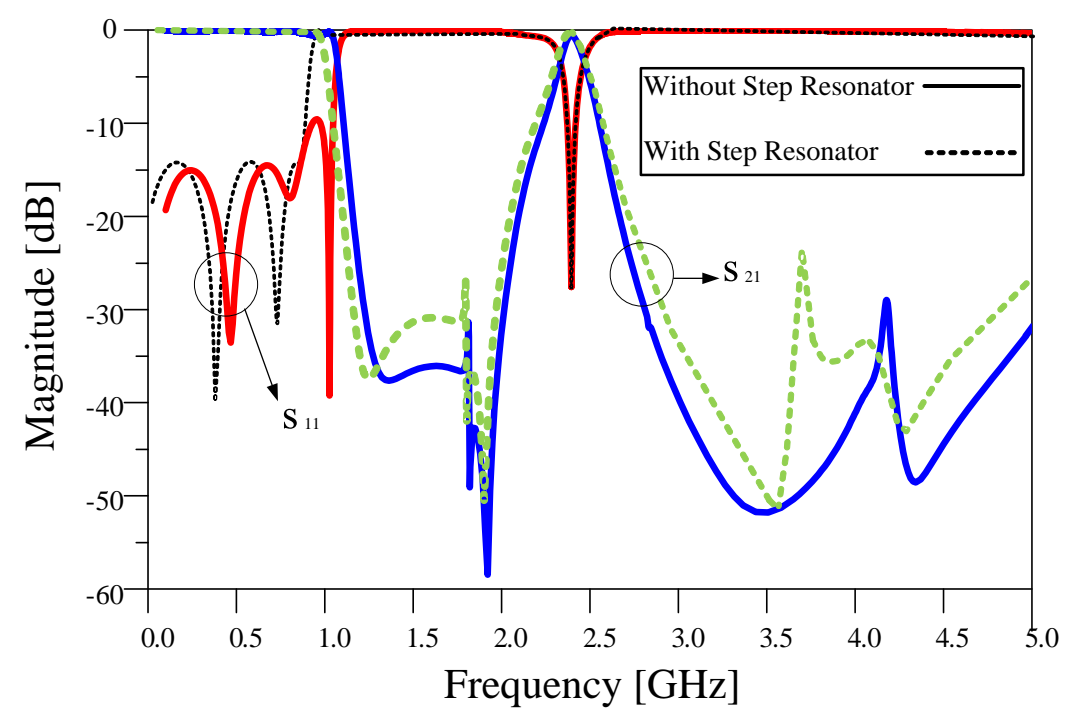

Figure 13. Frequency response of the proposed filter 
Table 2. Dimensions of the utilized transmission lines in the proposed filter.

\begin{tabular}{cccccccc}
\hline Par. $^{\text {a }}$ & Val. $^{\text {b }}$ & Par. & Val. & Par. & Val. & Par. & Val. \\
\hline L1 & 11.3 & L10 & 0.22 & W1 & 2 & W10 & 0.53 \\
\hline L2 & 21.1 & L11 & 6.4 & W2 & 2 & W11 & 1.5 \\
\hline L3 & 6.4 & L12 & 11.8 & W3 & 9.3 & W12 & 2.4 \\
\hline L4 & 5.4 & L13 & 9 & W4 & 8.6 & W13 & 1.2 \\
\hline L5 & 6.6 & L14 & 4.5 & W5 & 0.25 & W14 & 0.1 \\
\hline L6 & 10.3 & L15 & 11.4 & W6 & 4.6 & W15 & 0.4 \\
\hline L7 & 6.4 & L16 & 2.6 & W7 & 10.3 & W16 & 1.3 \\
\hline L8 & 12.3 & L17 & 19.4 & W8 & 0.1 & Wx, g1 & $0.7,0.2$ \\
\hline L9 & 0.24 & L18 & 6 & W9 & 0.18 & g2, g3 & $0.18,0.26$ \\
\hline
\end{tabular}

a. Parameter; $b$. Value

After combining the lowpass and bandpass filter the insertion loss parameter and impedance matching could not be achieved. Therefore, a stepped impedance resonator is added in the designed filter structure, which is shown with dimensions of W15 and L18 in Figure 12. The effect of this stepped impedance resonator on the proposed filter frequency response is depicted in Figure 13.

\section{Results of the Designed Dual Band Filter}

The gap (g3) between the coupled resonator of the bandpass filter can be used to justify the fundamental bandpass frequency and insertion loss of the band pass filter. Another important transmission line in the designed filter is shown with $\mathrm{W}_{\mathrm{X}}$ width in the layout of the designed filter. Also, the effects of the layout key parameters (g3 and $\mathrm{W}_{\mathrm{x}}$ ) on frequency response of the proposed filter are depicted in Figures 14 (a), (b), and (c). Besides, the effects of the layout key parameters (g3 and $\mathrm{W}_{\mathrm{X}}$ ) on lowpass fundamental frequency and insertion loss of the BPF are listed in Table 2. 
Saeed Roshani et al.

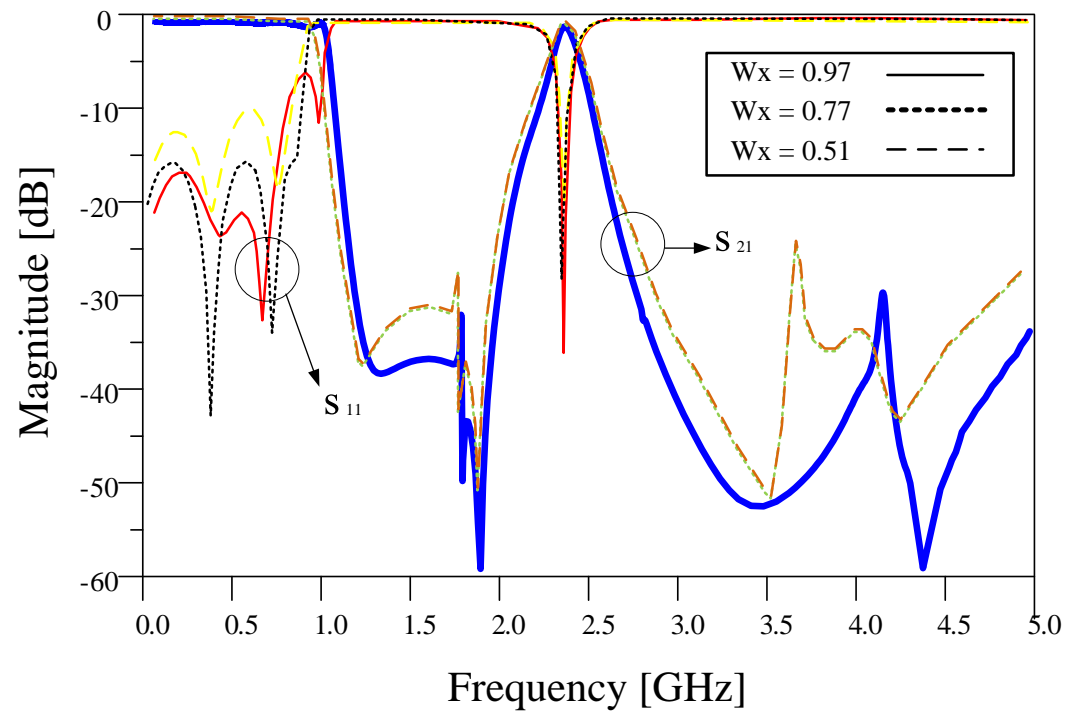

(a)

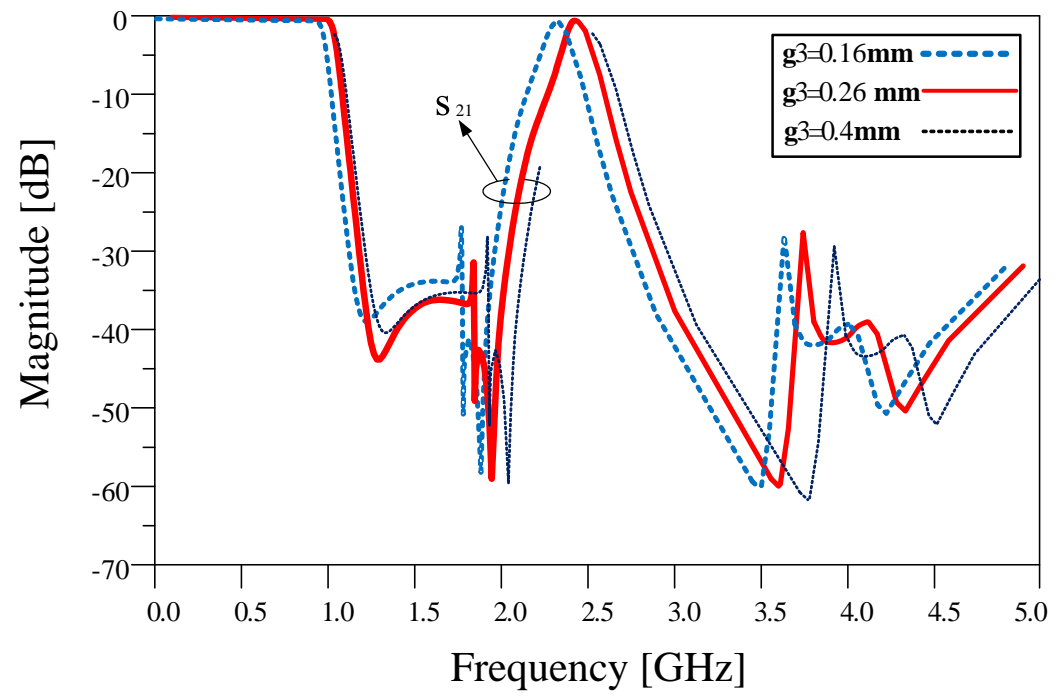

(b) 


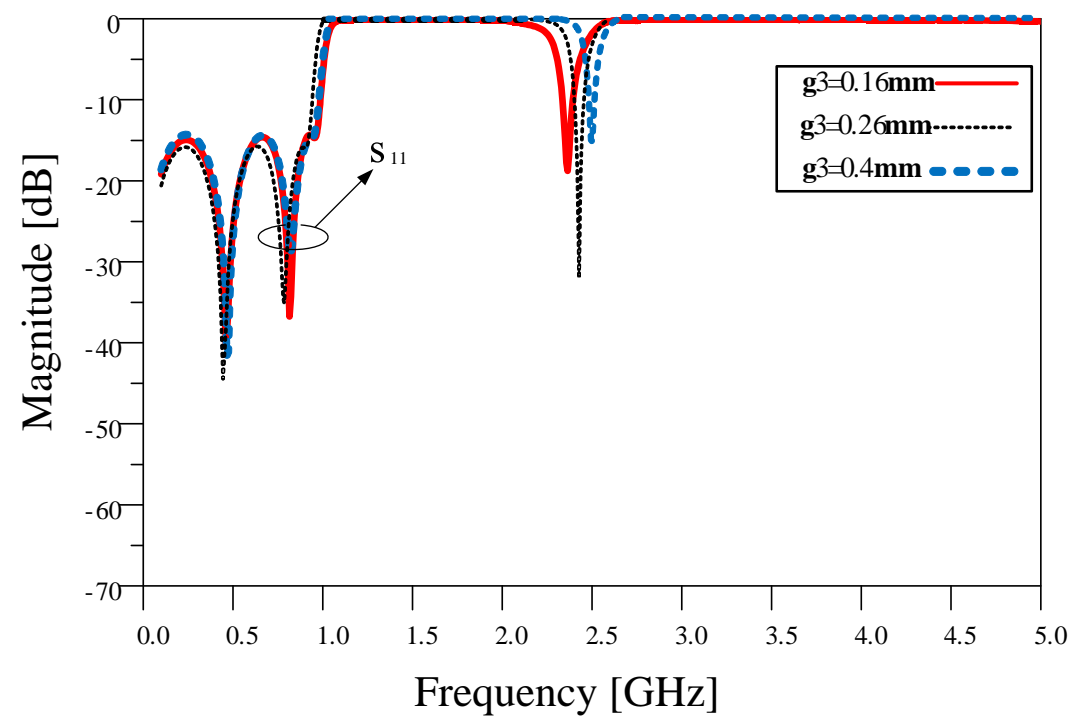

(c)

Figure 14. The effects of the layout key parameters ( $\mathrm{g} 3$ and $\mathrm{W}_{\mathrm{X}}$ ) on frequency response of the proposed filter. (a) the effect of $\mathrm{W}_{\mathrm{X}}$ on frequency response. (b) The effect of g3 on S21 and (c) S11 scattering parameters of the deigned dual band filter.

Table 3. Effects of the layout key parameters (g3 and $\mathrm{W}_{\mathrm{X}}$ ) on lowpass fundamental frequency and insertion loss of the BPF.

\begin{tabular}{ccc}
\hline g3 & $f_{0}$ (bandpass channel) & $\begin{array}{c}\text { Insertion loss of } \\
\text { bandpass }\end{array}$ \\
\hline $0.4 \mathrm{~mm}$ & $2.5 \mathrm{GHZ}$ & $1.25 \mathrm{~dB}$ \\
\hline $0.26 \mathrm{~mm}$ & $2.4 \mathrm{GHZ}$ & $0.58 \mathrm{~dB}$ \\
\hline $0.16 \mathrm{~mm}$ & $2.3 \mathrm{GHZ}$ & $0.58 \mathrm{~dB}$ \\
\hline
\end{tabular}

As results show, the fundamental frequency of $2.4 \mathrm{GHz}$ and the $1 \mathrm{GHz}$ cutoff frequency are achieved for the bandpass and lowpass channels, respectively. The obtained insertion losses are less than $0.5 \mathrm{~dB}$ and $0.2 \mathrm{~dB}$ for the bandpass and lowpass channels, respectively. The achieved return losses are better than $14 \mathrm{~dB}$ for lowpass channel and better than $28 \mathrm{~dB}$ for bandpass channel.

\section{Conclusion}

In this paper a dual band lowpass bandpass filter is designed by using the artificial neural network. Simple resonators and matching circuit is used for designing of the filter. The extracting of the transfer function is a complex procedure even impossible in some cases. Therefore, ANN and DL tools are used to model the 
Saeed Roshani et al.

transfer function of the LC circuit of the primary resonator. By achieved desirable results, this multi band filter can be utilized in modern wireless systems. The proposed filter enables the ability of having a lowpass filter and a bandpass filter in a single device.

\section{Conflicts of Interest}

No potential conflict of interest was reported by the authors.

\section{References}

[1] Roshani, .S and Roshani, S. (2019) Design of a very compact and sharp bandpass diplexer with bended lines for GSM and LTE applications. AEU-International Journal of Electronics and Communications. 1; 99:354-360.

[2] Bavandpour, S.K., Roshani, S., Pirasteh, A., Roshani, S. and Seyedi, H. (2021) A compact lowpass-dual bandpass diplexer with high output ports isolation. AEU-International Journal of Electronics and Communications. 1; 135:153748.

[3] Roshani, S., Roshani, S. and Zarinitabar, A. (2019) A modified Wilkinson power divider with ultra harmonic suppression using open stubs and lowpass filters. Analog Integrated Circuits and Signal Processing. 98(2):395-399.

[4] Jamshidi, M., Lalbakhsh, A., Lotfi, S., Siahkamari, H., Mohamadzade, B. and Jalilian, J. (2020) A neuro-based approach to designing a Wilkinson power divider. International Journal of $R F$ and Microwave Computer-Aided Engineering. 30(3):e22091.

[5] Pirasteh, A., Roshani, S. and Roshani, S. (2018) A modified class-F power amplifier with miniaturized harmonic control circuit. AEU-International Journal of Electronics and Communications. 1; 97:202-209.

[6] Nouri, M.E., Roshani, S., Mozaffari, M.H. and Nosratpour, A. (2020) Design of high-efficiency compact Doherty power amplifier with harmonics suppression and wide operation frequency band. AEU-International Journal of Electronics and Communications. 1; 118:153168.

[7] Chuang, M.L. and Wu, M.T. (2011) Microstrip diplexer design using common T-shaped resonator. IEEE Microwave and Wireless components letters. 6; 21(11):583-585.

[8] Deng, P.H., Tsai, J.T. and Liu, R.C. (2014) Design of a switchable microstrip dual-band lowpass-bandpass filter. IEEE Microwave and Wireless Components Letters. 11; 24(9):599-601.

[9] Xu, J. (2015) Compact microstrip tri-band lowpass-bandpass filter. Electronics Letters. 2; 51(19):1509-1510.

[10] Chen, F.C., Qiu, J.M., Hu, H.T., Chu, Q.X. and Lancaster, M.J. (2015) Design of microstrip lowpass-bandpass triplexer with high isolation. IEEE Microwave and Wireless Components Letters. 11; 25(12):805-807.

[11] Sattari, M.A., Roshani, G.H., Hanus, R. and Nazemi, E. (2021) Applicability of time-domain feature extraction methods and artificial intelligence in 
two-phase flow meters based on gamma-ray absorption technique. Measurement, 168, 108474, doi:10.1016/j.measurement.2020.108474.

[12] Roshani, G., Hanus, R., Khazaei, A., Zych, M., Nazemi, E. and Mosorov, V. (2018) Density and velocity determination for single-phase flow based on radiotracer technique and neural networks. Flow Meas. Instrum., 61, 9-14, doi:10.1016/j.flowmeasinst.2018.03.006.

[13] Karami, A., Roshani, G.H., Khazaei, A., Nazemi, E. and Fallahi, M. (2018) Investigation of different sources in order to optimize the nuclear metering system of gas-oil-water annular flows. Neural Comput. Appl., 32, 3619-3631, doi: 10.1007/s00521-018-3673-0.

[14] Karami, A., Roshani, G.H., Nazemi, E. and Roshani, S. (2018) Enhancing the performance of a dual-energy gamma ray based three-phase flow meter with the help of grey wolf optimization algorithm. Flow Meas. Instrum, 64, 164-172

[15] Nazemi E., Roshani G.H., Feghhi S.A.H., Setayeshi S., Eftekhari Zadeh E. and Fatehi A. (2016) Optimization of a method for identifying the flow regime and measuring void fraction in a broad beam gamma-ray attenuation technique, international journal of hydrogen energy, 41, 7438-7444.

[16] Jamshidi, M.B., Lalbakhsh, A., Mohamadzade, B., Siahkamari, H., Mousavi, S.M. (2019) A novel neural-based approach for design of microstrip filters. AEU-International Journal of Electronics and Communications. 1; 110:152847.

[17] Heshmati, H., Roshani, S. (2018) A miniaturized lowpass bandpass diplexer with high isolation. AEU-International Journal of Electronics and Communications. 1; 87:87-94. 